\title{
Properties of the Molecular Clump and the Associated Ultracompact H it REgion in THE GAS SHELl OF THE EXPANDING H II REGION SH 2-104
}

\author{
Young Chol Minh ${ }^{1}$, Kee-Tae Kim ${ }^{1}$, Chi-Hung Yan ${ }^{2,3}$, Yong-Sun Park ${ }^{4}$, Seokho Lee ${ }^{4,5}$, Dharam Vil Lal ${ }^{6}$, \\ Tatsuhiko Hasegawa ${ }^{2}$, X. Z. Zhang ${ }^{7}$, And Yi-Jeng KuAn ${ }^{3}$ \\ ${ }^{1}$ Korea Astronomy and Space Science Institute, 776 Daeduk-daero, Yuseong, Daejeon 305-348, Korea; minh@kasi.re.kr \\ ${ }^{2}$ Academia Sinica Institute of Astronomy and Astrophysics, P.O. Box 23-141, Taipei 106, Taiwan \\ ${ }^{3}$ Department of Earth Sciences, National Taiwan Normal University, Taipei 10617, Taiwan \\ ${ }^{4}$ Astronomy Program, Department of Physics and Astronomy, Seoul National University, Gwanak, Seoul 151-747, Korea \\ ${ }^{5}$ Department of Astronomy and Space Science, Kyung Hee University, Yongin-shi, Kyungki-do 446-701, Korea \\ ${ }^{6}$ National Centre for Radio Astrophysics, Tata Institute of Fundamental Research, Pune University Campus, Post Bag 3 , \\ Ganeshkhind, Pune - 411007, India \\ ${ }^{7}$ National Astronomical Observatories of China, Chinese Academy of Sciences, 20A Datun Road, Chaoyang District, \\ Beijing 100012, China
}

Received July 3, 2014; accepted Septembr 22, 2014

\begin{abstract}
We study the physical and chemical properties of the molecular clump hosting a young stellar cluster, IRAS 20160+3636, which is believed to have formed via the "collect and collapse" process. Physical parameters of the UC H II region associated with the embedded cluster are measured from the radio continuum observations. This source is found to be a typical Galactic UC H II region, with a B0.5 type exciting star, if it is ionized by a single star. We derive a $\mathrm{CN} / \mathrm{HCN}$ abundance ratio larger than 1 over this region, which may suggest that this clump is being affected by the UV radiation from the HII region.
\end{abstract}

Key words: ISM: abundances — ISM: molecules — ISM: HII regions — individual: Sh 2-104

\section{INTRODUCTION}

Young stars sometimes form at the periphery of $\mathrm{HII}$ regions. In some cases the expansion of $\mathrm{H}$ II regions is thought to have triggered the star formation in the fragmented clumps in various ways as summarized by Deharveng et al. (2005). Among these processes, the "collect and collapse" method is believed to have formed the embedded cluster, IRAS 20160+3636, in the molecular ring around the HII region Sharpless 104 (Sh 2-104: hereafter Sh104, Deharveng et al. 2003). In this process the expanding H II region sweeps up the surrounding neutral gas and generates the dense, fragmented shocked layer, which leads to massive star formation (Elmegreen \& Lada 1977; Whitworth et al. 1994). Deharveng et al. (2003) have analyzed the Sh104 region, especially on the molecular ring around the H II region, as a typical example of the collect and collapse process. Here we report the properties of the molecular clump embedding IRAS $20160+3636$ and the associated ultracompact (UC) H II region at the eastern periphery of the ring-like shell around Sh104.

Sh104 is an optically visible Galactic H II region located in the Cygnus region. This diffuse $\mathrm{H}$ II region, excited by an O6V star (Israël 1977; Crampton et al. 1978; Lahulla 1985), is surrounded by a circularly shaped molecular gas ring, probably formed by the expansion of the H II region. The distance to this source was first de-

Corresponding author: Y. C. Minh termined kinematically to be about $5.3 \mathrm{kpc}$ by measuring the velocity of the $\mathrm{H} \alpha$ line (Georgelin et al. 1973). The distance to the diffuse $\mathrm{H}$ II region was estimated to be $5 \mathrm{kpc}$ by Israël (1977) from the distance modulus of the exciting star, and $4.8 \mathrm{kpc}$ by Lahulla (1985) from UBVRI photometry of stars in this region. Deharveng et al. (2003) used $4 \mathrm{kpc}$ as the distance to this source. We also applied the $4 \mathrm{kpc}$ as the distance to this source.

Using the data taken at $1 \mathrm{GHz}$ with GMRT (Giant Metrewave Radio Telescope), and at $3 \mathrm{~mm}$ and $1 \mathrm{~mm}$ with ARO (Arizona Radio Observatory) telescopes, we derived physical properties of the associated UC H II region and the molecular clump embedding this stellar cluster. The observations and data used are included in Section 2, observational results and discussions in Section 3, and the summary in Section 4.

\section{Observational Data}

\subsection{ARO Observations}

The millimeter-wave molecular line data were taken in 2006 May using the $12 \mathrm{~m}$ telescope at Kitt Peak and the Submillimeter Telescope (SMT) ${ }^{\mathbf{1}}$ at Mt. Graham, Arizona. The receivers used were dual-channel, cooled SIS mixers operated in single-sideband dual polarization mode with image rejection of at least $16 \mathrm{~dB}$. The

\footnotetext{
$\mathbf{1}_{\text {These telescopes are operated by the Arizona Radio Observa- }}$ tory (ARO), Steward Observatory, University of Arizona, with partial support from the Research Corporation.
} 
Table 1

Molecular line observational parameters

\begin{tabular}{cccccc}
\hline Molecule & Transition & $\begin{array}{c}\text { Frequency } \\
(\mathrm{GHz})\end{array}$ & $\begin{array}{c}\mathrm{T}_{\text {sys }}^{\mathrm{b}} \\
(\mathrm{K})\end{array}$ & $\begin{array}{c}r m s(1 \sigma)^{\mathrm{c}} \\
(\mathrm{K})\end{array}$ & Telescope \\
\hline${ }^{12} \mathrm{CO}$ & $\mathrm{J}=1-0$ & 115.2712 & $450-700$ & $0.25-0.32$ & $12 \mathrm{~m}$ \\
$\mathrm{C}^{18} \mathrm{O}$ & $\mathrm{J}=1-0$ & 109.7822 & $200-230$ & $0.06-0.07$ & $12 \mathrm{~m}$ \\
$\mathrm{CN}$ & $\mathrm{N}=1-0$ & 113.4910 & $250-300$ & $0.07-0.08$ & $12 \mathrm{~m}$ \\
& $\mathrm{~N}=2-1$ & 226.6595 & $390-400$ & $\sim 0.04$ & $\mathrm{SMT}$ \\
$\mathrm{HCN}$ & $\mathrm{J}=1-0$ & 88.6318 & $250-290$ & $0.04-0.05$ & $\mathrm{SMT}$ \\
& $\mathrm{J}=3-2$ & 265.8864 & $460-470$ & $\sim 0.05$ & $\mathrm{SMT}$ \\
\hline
\end{tabular}

${ }^{a}$ From NIST Standard Reference Database (F. J. Lovas 2003, http://physics.nist.gov/PhysRefData). ${ }^{b}$ Observed system temperatures. ${ }^{\mathrm{C}}$ Observed spectral $\mathrm{rms}(1 \sigma)$ noise.

backends used for the observations were 256 channel filter banks of $500 \mathrm{kHz}$ and $1 \mathrm{MHz}$ resolution operating in parallel mode. The different polarization data were averaged together to improve the spectral $\mathrm{rms}$ noise. Pointing was calibrated by observations of planets, Saturn and Jupiter. The field center of all observations was $(\alpha, \delta)_{J 2000}=\left(20^{h} 17^{m} 45^{s}, 36^{\circ} 46^{\prime} 00^{\prime \prime}\right)$ with the systemic velocity of this source $\mathrm{v}_{\text {lsr }}=0 \mathrm{~km}$ $\mathrm{s}^{-1}$ (cf., Israël 1977). The maps were made in two different grids: (1) for the ${ }^{12} \mathrm{CO}(1-0), \mathrm{C}^{18} \mathrm{O}(1-0)$, $\mathrm{HCN}(1-0)$, and $\mathrm{CN}(1-0)$ transitions, in the offset range $+1^{\prime} \leq \Delta \alpha \leq+5^{\prime}$ and $-3^{\prime} \leq \Delta \delta \leq+1^{\prime}$ from the center position by $1^{\prime}$ spacings, and (2) for the HCN $(3-2)$ and $\mathrm{CN}(2-1)$ transitions, in the offset range $+0.5^{\prime} \leq \Delta \alpha \leq+3.5^{\prime}$ and $-2.5^{\prime} \leq \Delta \delta \leq+0.5^{\prime}$ from the center position by $0.5^{\prime}$ spacings. The data were taken in position-switching mode with the off position $(\alpha, \delta)_{J 2000}=\left(20^{h} 18^{m} 10^{s}, 36^{\circ} 44^{\prime} 20^{\prime \prime}\right)$. The beam sizes are about 65 " and 32 " at $100 \mathrm{GHz}(12 \mathrm{~m})$ and $240 \mathrm{GHz}$ (SMT), respectively. For the data reduction and map images, the GILDAS (Grenoble Image and Line Data Analysis System) software package ${ }^{2}$ was used.

The observed molecular transitions and system parameters are listed in Table $1 .{ }^{12} \mathrm{CO}$ is known to be sensitive to the kinetic temperature of the cloud because of its low dipole moment (0.1 Debye) and high optical depth, but its rare isotope variant $\mathrm{C}^{18} \mathrm{O}$ is thought to trace gas density in the region of about $\mathrm{n}_{\mathrm{H}_{2}} \gtrsim 10^{3}$ $\mathrm{cm}^{-3}$. HCN is commonly used as a dense gas tracer for densities $\mathrm{n}_{\mathrm{H}_{2}} \gtrsim 10^{4} \mathrm{~cm}^{-3}$ and $\mathrm{CN}$ is also another tracer of dense gas, with a lower (by a factor of 5 ) critical density than HCN (cf., Pérez-Beaupuits et al. 2007). The temperature scale was calibrated to the radiation temperature $\mathrm{T}_{\mathrm{R}}^{*}$ for the $12 \mathrm{~m}$ data and the antenna temperature scale, $\mathrm{T}_{\mathrm{A}}^{*}$ for the SMT data. Assuming the source fills the main beam, the main-beam temperature is estimated to be $\mathrm{T}_{\mathrm{R}}=\mathrm{T}_{\mathrm{R}}^{*} / \eta_{\mathrm{c}}$ and $\mathrm{T}_{\mathrm{R}}=\mathrm{T}_{\mathrm{A}}^{*} / \eta_{\mathrm{mb}}$ for the $12 \mathrm{~m}$ and SMT data, respectively, where the coupling efficiencies are taken to be $\eta_{\mathrm{c}} \sim 0.9$ at $100 \mathrm{GHz}$ and $\eta_{\mathrm{mb}} \sim 0.7$ at around $240 \mathrm{GHz}^{\mathbf{3}}$

\footnotetext{
$\mathbf{2}_{\text {http://www.iram.fr/IRAMFR/GILDAS }}$

${ }^{3}$ See the ARO web-site http://aro.as.arizona.edu for details
}

\subsection{GMRT Observations}

The ionized gas toward Sh104 has been mapped at 1.06 $\mathrm{GHz}$ with a bandwidth of $8 \mathrm{MHz}$ in 2003 January using the Giant Metrewave Radio Telescope (GMRT) array in Pune, India. The on-source integration time is about $70-80$ minutes. The GMRT consists of 30 fully steerable dishes, of 45 meter diameter with a maximum baseline of $\sim 25 \mathrm{~km}$ (Swarup et al. 1991). The aperture efficiency of the dishes is $\sim 40 \%$ in the $1 \mathrm{GHz}$ band, which implies an effective collecting area of $\sim 19000$ $\mathrm{m}^{2}$. The receiver is a prime focus uncooled receiver with a characteristic system temperature of $\sim 70 \mathrm{~K}$. The observations were made in the standard spectral line mode with a spectral resolution of $125 \mathrm{kHz}$. The sources 3C 48 and $2355+498$ were used as the primary flux density and the secondary phase calibrators, respectively. The visibility data were converted to FITS and analyzed using classic AIPS $^{4}$ in the standard way. In addition to normal editing of the data, we identified and flagged time ranges affected by scintillation and channels affected by radio frequency interference, after which the central channels were averaged using the AIPS task 'SPLAT' to reduce the data volume. We used the AIPS task 'IMAGR' to map the full field at this frequency. To obtain the high-resolution image that is also sensitive to extended structure, we employed the SDI CLEANing algorithm (Steer et al. 1984). We used uniform weighting and the 3D option for $w$-term correction throughout our analysis. The presence of a large number of point sources in the field allowed us to do phase self-calibration to improve the image. At each iteration of self-calibration, the Fourier transform of the image and the visibilities were compared to check for improvement in the source model.

Figure 1 shows the observed radio continuum image of Sh104 at low- and high-resolution with GMRT, as described in the figure caption. The high resolution map used the full UV data (corresponds to $\sim 0.16-100$ kilo-lambda; giving a resolution of $\sim 2$ arcsec), whereas the low-resolution map was made after tapering $(\sim 0.16-10$ kilo-lambda; giving a resolution of $\sim 21$ arcsec) the full UV-data. The primary field of view at $1.06 \mathrm{GHz}$ is about $0.4^{\circ}$. This image has an

\footnotetext{
${ }^{4}$ Astronomical Image Processing System; http://www.aips. nrao.edu
} 

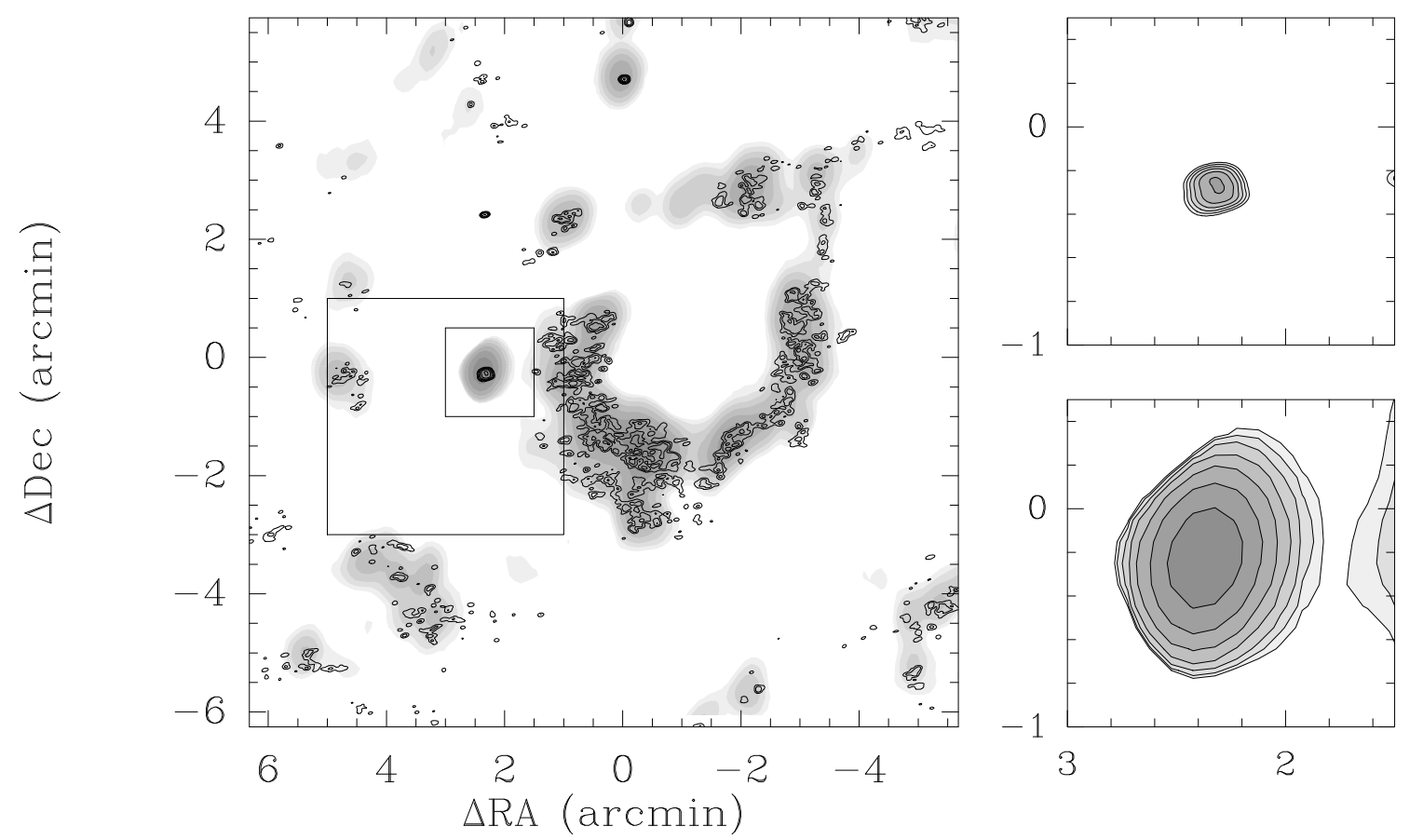

Figure 1. (Left) The $1.06 \mathrm{GHz}$ continuum intensity map obtained toward Sh104 with GMRT. The $\left(0^{\prime}, 0^{\prime}\right)$ position of this map is $(\alpha, \delta)_{J 2000}=\left(20^{h} 17^{m} 45^{s}, 36^{\circ} 46^{\prime} 00^{\prime \prime}\right)$. The low-resolution $\left(\sim 31^{\prime \prime} \times 21^{\prime \prime} ;\right.$ P.A. $\left.=82^{\circ}\right)$ result is in grey scale and the high-resolution $\left(\sim 5^{\prime \prime} \times 3^{\prime \prime}\right.$; P.A. $\left.=33^{\circ}\right)$ in contours. Both grey and contour scales start at $3 \times 10^{-4} \mathrm{Jy} \mathrm{beam}^{-1}$, and increase by a factor of 2 for next step. The peak intensities are $3.24 \times 10^{-2}$ and $1.06 \times 10^{-2} \mathrm{Jy}_{\text {beam }}{ }^{-1}$ for low- and highresolution results, respectively. The continuum source near the offset $\left(\sim 2.3^{\prime},-0.3^{\prime}\right)$ coincides with the $I R A S$ point source IRAS $20160+3636$. The larger square box shows the region where molecular lines are observed using the ARO telescopes. The smaller square box is the region expanded in the right side: for the high-resolution (right upper) and the low-resolution (right lower). Contour levels are same as those in the left panel.

rms of $\sim 0.13$ mJy beam ${ }^{-1}$. The flux density uncertainly is less than a 2 percent, which is smaller than the rms in the image. The properties of the extended diffuse H II region have been well investigated by previous studies, (e.g., Reifenstein et al. 1970; Georgelin et al. 1973; Israël 1977; Lahulla 1985). Here we focus on the UC H II region and the dense molecular clump associated with IRAS $20160+3636$.

\section{RESUltS AND DISCUSSION}

\subsection{The Ultracompact $H$ II Region}

An UC Hil region has been found in association with a young stellar cluster, IRAS $20160+3636$, deeply embedded in the dense molecular gas (Condon et al. 1998). The UC H II region is marginally resolved in our highresolution $\left(5^{\prime \prime} \times 3^{\prime \prime}\right.$; P.A. $\left.=33^{\circ}\right)$ map and looks spherical (Figure 1), but unresolved in our low-resolution $\left(31^{\prime \prime} \times 21^{\prime \prime} ;\right.$ P.A. $\left.=82^{\circ}\right)$ map. The size at half maximum was determined by two-dimensional Gaussian fitting for the high resolution result. The deconvolved size $7.0^{\prime \prime} \times 6.0^{\prime \prime}$ corresponds to $0.14 \mathrm{pc} \times 0.12 \mathrm{pc}$, at a distance of $4 \mathrm{kpc}$ to this source. Kim \& Koo (2001) found that the vast majority of UC H II regions have diffuse extended envelopes and suggested that they are not real ionization-bounded UC H II regions, but compact cores of more extended H II regions (see also Kurtz et al. 1999; Ellingsen et al. 2005). In this context, the UC
H II region associated with IRAS $20160+3636$ seems to be a bona-fide UC H II region and younger than the UC $\mathrm{H}$ II regions with extended envelopes. Wood \& Churchwell (1989) identified 1646 IRAS point sources as UC $\mathrm{H}$ II region candidates using IRAS color criteria, and argued that $10 \%-20 \%$ of massive stars are in the UC H II region phase. This implies that their ages may be $10 \%-20 \%$ of the typical main-sequence lifetime of massive stars, $\gtrsim 1 \times 10^{5}$ yr. Later many of them were found to have extended envelopes with sizes $>1^{\prime}$ (Kurtz et al. 1999; Kim \& Koo 2001). For comparison, the UC H II region of IRAS $20160+3636$ is at a dynamic age of $\sim 1 \times 10^{4} \mathrm{yr}$, assuming an expansion velocity of $10 \mathrm{~km} \mathrm{~s}^{-1}$.

From the $1.06 \mathrm{GHz}$ radio continuum data, we derived the physical parameters of the UC H II region using the formulae of Mezger \& Henderson (1967) and listed in Table 2. We assumed that the H II region is spherically symmetric, homogeneous, optically thin, dust-free, and ionization-bounded, and that the electron temperature is $10^{4} \mathrm{~K}$. The derived physical parameters are similar to those of other UC HII regions except electron density and emission measure, which are signficantly smaller than the typical values, $n_{\mathrm{e}}>10^{4} \mathrm{~cm}^{-3}$ and $\mathrm{EM}>10^{6} \mathrm{pc} \mathrm{cm}^{-6}$. This may originate from the assumption that the observed $1.06 \mathrm{GHz}$ continuum emission is optically thin. UC H II regions can easily get optically thick around $1 \mathrm{GHz}$ and so the previous ra- 

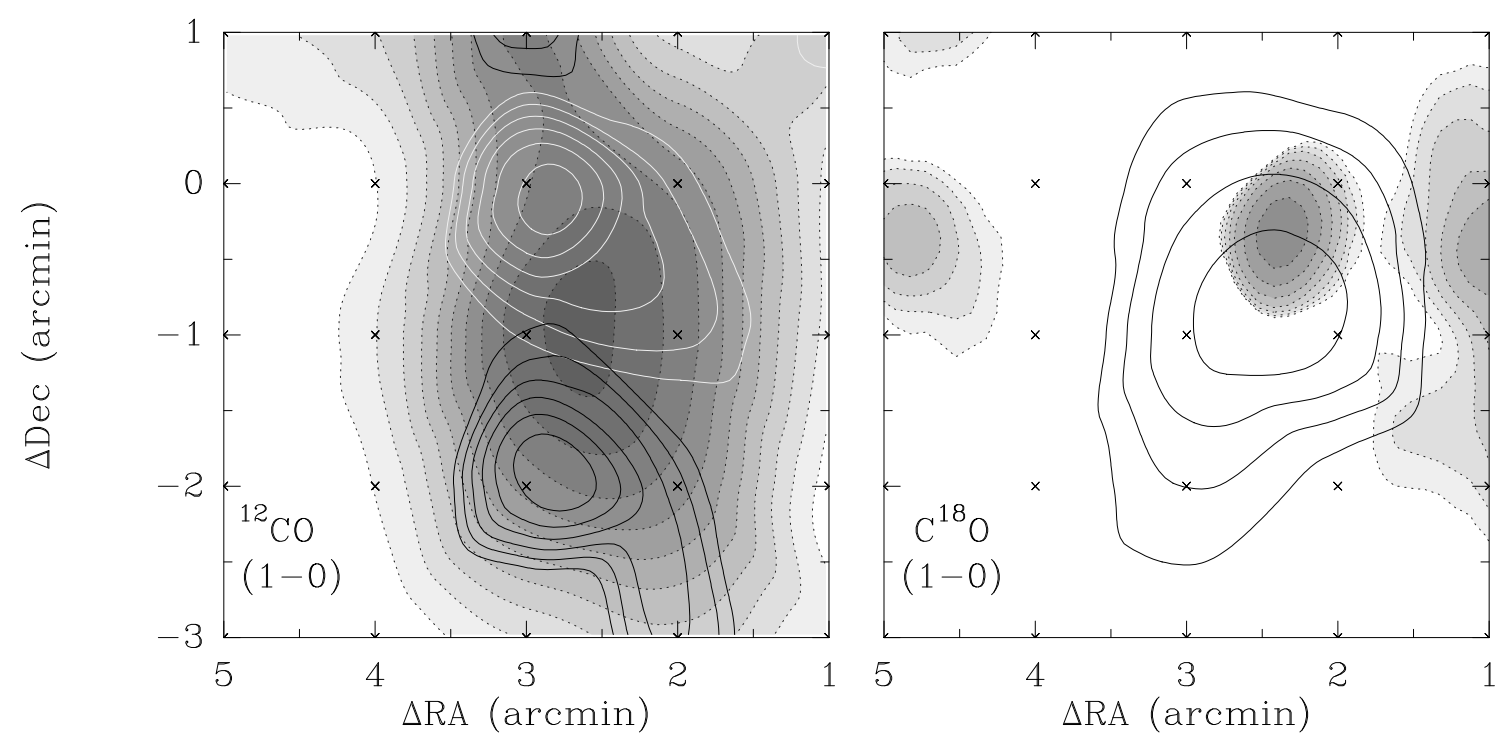

Figure 2. Velocity integrated intensity $\left(\int \mathrm{T}_{\mathrm{R}}^{*} \mathrm{~d} v\right)$ map of the ${ }^{12} \mathrm{CO}(1-0)$ line (left, in grey) and the $\mathrm{C}^{18} \mathrm{O}(1-0)$ line $($ right, in solid contours). Crosses are observed positions. (Left) Contour levels of the ${ }^{12} \mathrm{CO}(1-0)$ line increase by $5 \mathrm{~K} \mathrm{~km} \mathrm{~s}$ from $5 \mathrm{~K} \mathrm{~km} \mathrm{~s}^{-1}$. High velocity components are shown with white and black solid contours, integrated for $v=-4 \sim-2 \mathrm{~km}$ $\mathrm{s}^{-1}$ and $2 \sim 4 \mathrm{~km} \mathrm{~s}^{-1}$, respectively. Contour levels for both high velocity components increase by $0.5 \mathrm{~K} \mathrm{~km} \mathrm{~s}^{-1}$ from $4 \mathrm{~K}$ $\mathrm{km} \mathrm{s}^{-1}$. (Right) Contour levels of the $\mathrm{C}^{18} \mathrm{O}(1-0)$ line increase by $0.4 \mathrm{~K} \mathrm{~km} \mathrm{~s}^{-1}$ from $0.2 \mathrm{~K} \mathrm{~km} \mathrm{~s}^{-1}$. The $1 \mathrm{GHz}$ continuum intensity in Figure 1 is shown in grey scale together with dotted contours.

dio continuum observations of them have been usually carried out at $\geq 5 \mathrm{GHz}$ (Wood \& Churchwell 1989; Kurtz et al. 1994). If the UC H II region is optically thick at $<5 \mathrm{GHz}$ as many other UC H II regions are, e.g., G5.89-0.39 (Gomez et al. 1991), the flux density would be proportional to the frequency squared. In that case, the $5 \mathrm{GHz}$ flux density is about 25 times larger than the $1.06 \mathrm{GHz}$ flux density and one can obtain electron density of $\sim 1 \times 10^{4} \mathrm{~cm}^{-3}$ and emission measure of $\sim 1 \times 10^{6} \mathrm{pc} \mathrm{cm}^{-6}$. We also evaluated the Lyman continuum photon flux under the assumption that the UC H II region is produced by a single ionizing star (Rubin 1968). If a stellar cluster is responsible for the ionization, the most massive star in the cluster may be 2 subclasses later-type than the single-star spectral type equivalent (see Table 7 of Kurtz et al. 1994).

\subsection{The Molecular Cloud Associated with the UC H II Region}

Figures 2 and 3 show integrated intensity maps of the observed transitions in Table 1 obtained toward the region indicated as a larger square box of the left panel in Figure 1. The molecular cloud hosting IRAS $20160+3636$ has a well defined boundary with a simple geometry, isolated from the background gas components. The ${ }^{12} \mathrm{CO}(1-0)$ line emission is a little more extended than those of the $\mathrm{CN}(1-0)$ and $\mathrm{HCN}$ $(1-0)$ lines, but they all show similar simple morphologies elongated in the north-south direction. We found a dense molecular core near the UC Hil region within this cloud, traced by the $\mathrm{CN}(2-1)$ and $\mathrm{HCN}$ $(3-2)$ lines (Figure 3$)$. The dense gas is mainly associated with the $1 \mathrm{GHz}$ continuum emission feature, but extended structures exist toward the south direction.
Table 2

Parameters of the UC Hil region ${ }^{\text {a }}$

\begin{tabular}{|c|c|}
\hline Size $^{\mathrm{b}}$, observed (arcsec) & $\begin{array}{l}8.2^{\prime \prime} \times 7.6^{\prime \prime} \\
\left.\text { (P.A. }+79^{\circ}\right)\end{array}$ \\
\hline Size $^{\mathrm{b}}$, deconvolved (arcsec) & $\begin{array}{l}7.0^{\prime \prime} \times 6.0^{\prime \prime} \\
\left(\text { P.A. }+25^{\circ}\right)\end{array}$ \\
\hline Peak Flux Density (mJy beam ${ }^{-1}$ ) & 10.6 \\
\hline Integrated Flux Density (mJy) & 41.7 \\
\hline Electron Number Density, $n_{\mathrm{e}}\left(\mathrm{cm}^{-3}\right)$ & $1.4 \times 10^{3}$ \\
\hline Emission Measure, EM (pc cm $\left.{ }^{-6}\right)$ & $3.7 \times 10^{5}$ \\
\hline Excitation Parameter, $\mathrm{U}\left(\mathrm{pc} \mathrm{cm}^{-2}\right)$ & 11.7 \\
\hline Mass of the UC HII Region, $\mathrm{M}_{\mathrm{HII}}\left(\mathrm{M}_{\odot}\right)$ & 0.1 \\
\hline Sound Crossing Time (yr) & $1.9 \times 10^{4}$ \\
\hline Lyman Cont. Photon Flux, $\log \mathrm{N}_{\mathrm{c}}^{\prime}\left(\mathrm{s}^{-2}\right)$ & 46.71 \\
\hline Spectral Type of the Exciting Star & B0.5 \\
\hline
\end{tabular}

${ }^{a}$ Derived by assuming optically thin emission and the distance of $4 \mathrm{kpc}$. ${ }^{\mathrm{b}}$ Diameter at half-maximum determined by twodimensional Gaussian fitting.

As shown in Figure 4, the observed CO spectra clearly show velocity wing components around $v_{\mathrm{lsr}} \sim-3$ and $+3 \mathrm{~km} \mathrm{~s}^{-1}$. We included the high-velocity component maps in Figure 2 together with the ${ }^{12} \mathrm{CO}(1-0)$ map; they appear to be located along the north-south direction: the blue-component around $\Delta \delta=0$ and the red-component at $\Delta \delta=-2^{\prime}$. The aligned direction of the high-velocity component coincides with the general elongation of the molecular cloud. It is not clear, however, whether this component results from the embedded stellar cluster or the interaction with the expanding $\mathrm{H}$ II region. We found no significant peak velocity gradient over the whole cloud.

Sizes of the cloud and the dense core were estimated to be $\sim 4.0 \times 2.6 \mathrm{pc}(\mathrm{HPW})$ and $\sim 1.6 \times 1.2 \mathrm{pc}$ 

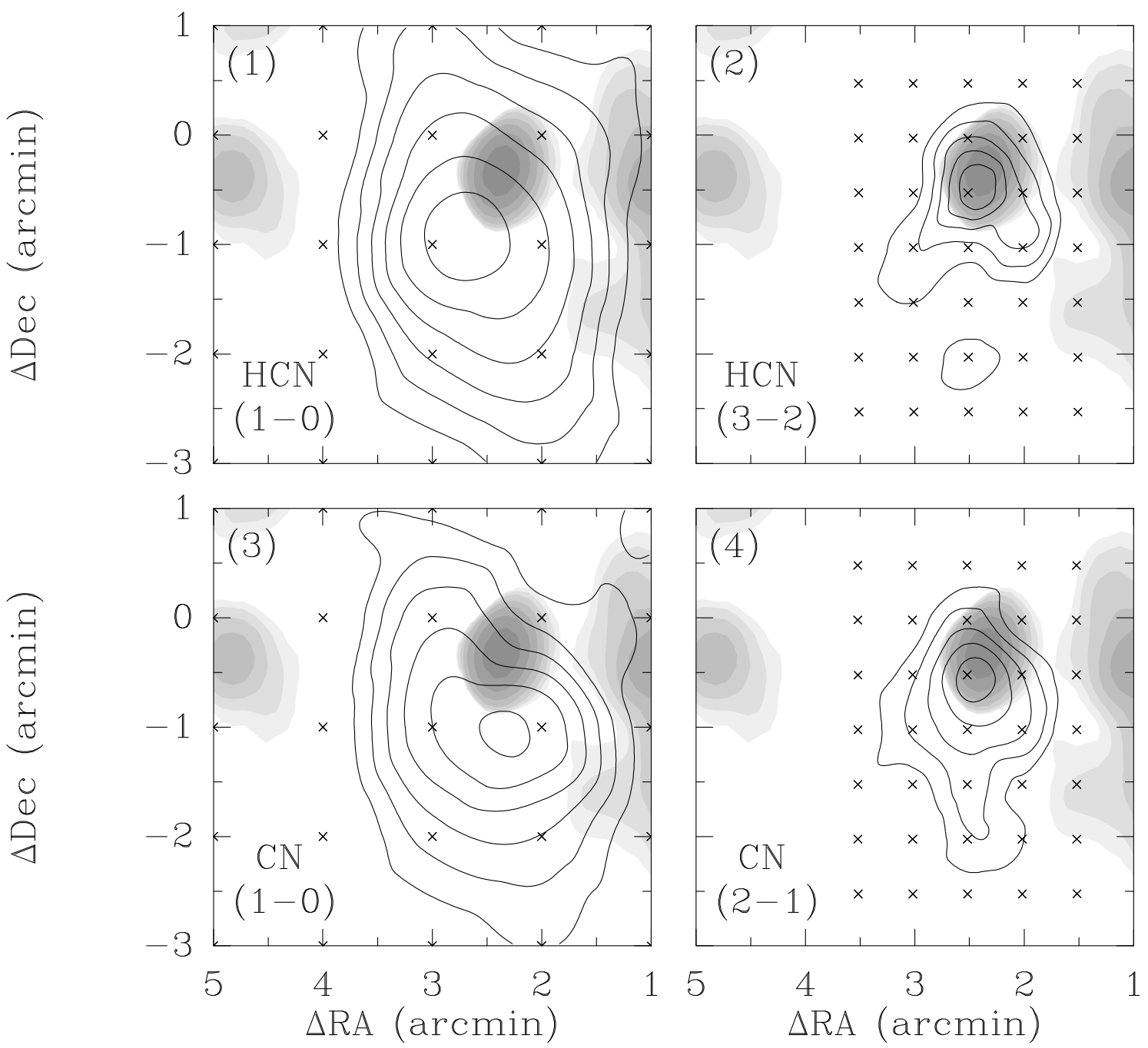

Figure 3. Velocity integrated intensity $\left(\int \mathrm{T}_{\mathrm{R}}^{*} \mathrm{~d} v\right)$ maps of the observed $\mathrm{HCN}$ and $\mathrm{CN}$ transitions as indicated in each panel. Crosses are observed positions. The $1 \mathrm{GHz}$ continuum intensity in Figure 1 is shown in grey scales. (1) HCN (1-0): Contour levels increase by $1 \mathrm{~K} \mathrm{~km} \mathrm{~s}^{-1}$ from $0.5 \mathrm{~K} \mathrm{~km} \mathrm{~s}^{-1}$. (2) $\mathrm{HCN}(3-2)$ : Contour levels increase by $0.2 \mathrm{~K} \mathrm{~km} \mathrm{~s}^{-1}$ from $0.2 \mathrm{~K} \mathrm{~km} \mathrm{~s}^{-1}$. (3) $\mathrm{CN}(1-0)$ : Contour levels increase by $0.5 \mathrm{~K} \mathrm{~km} \mathrm{~s}^{-1}$ from $0.3 \mathrm{~K} \mathrm{~km} \mathrm{~s}^{-1}$. (4) CN (2-1): Contour levels increase by $0.4 \mathrm{~K} \mathrm{~km} \mathrm{~s}^{-1}$ from $0.2 \mathrm{~K} \mathrm{~km} \mathrm{~s}^{-1}$.

(HPW), using the HCN $(1-0)$ and $(3-2)$ lines, respectively, assuming a distance of $4 \mathrm{kpc}$. We derived the total $\mathrm{C}^{18} \mathrm{O}$ column density, by assuming optically thin emission $\left(\tau_{\mathrm{C}^{18} \mathrm{O}(1-0)} \approx 0.03-0.05\right.$ by comparing the intensity with the $\mathrm{CO} 1-0$ line at peak, assuming the $\left.{ }^{16} \mathrm{O} /{ }^{18} \mathrm{O}=500\right)$ and an LTE condition, $\mathrm{N}_{\mathrm{C}^{18} \mathrm{O}}=1.6 \pm 0.3 \times 10^{15} \mathrm{~cm}^{-2}$ toward the offset $(+2,-1)$ position. The uncertainty of the column density is from the applied rotational temperature range, $\mathrm{T}_{\text {rot }}=15-20 \mathrm{~K}$ (Deharveng et al. 2003), together with a typical spectral $\mathrm{rms}$ noise $(1 \sigma)$ of $\sim 0.06-0.07 \mathrm{~K}$ of the observed spectra (Table 1 ). We used the relation $\mathrm{N}_{\mathrm{H}_{2}} \approx 0.5-1.0 \times 10^{7} \cdot \mathrm{N}_{\mathrm{C}^{18} \mathrm{O}}$ (see discussions and references in Harjunpää et al. 2004; Burgh et al. 2007) to derive the total hydrogen column density. From the estimated total hydrogen column densities we expected visual extinctions of $\mathrm{A}_{\mathrm{V}} \approx 1-3 \mathrm{mag}$ at the periphery of the cloud $\left(\mathrm{A}_{\mathrm{V}}=\mathrm{N}_{\mathrm{H}_{2}} / 0.94 \times 10^{21}\right.$, Frerking et al. 1982), and $7-20$ mag near the peak position, and the averaged $\mathrm{A}_{\mathrm{V}}$ of about 10 mag over the whole cloud.

Using the equation, $\mathrm{M}_{\mathrm{gas}}=2 \times \mathrm{N}_{\mathrm{H}_{2}} \times \mu \mathrm{m}_{\mathrm{H}} \times$ Area(inside of HPW), where $\mu$ is 1.4 amu per $\mathrm{H}$ nuclei and accounts for the mass of He and other elements and the 'Area' is estimated from the HPW $(4.0 \times 2.6 \mathrm{pc})$, we derived the total gas mass of the molecular cloud, $\mathrm{M}_{\text {total }} \sim(1-3) \times 10^{3} \mathrm{M}_{\odot}$.

\section{3. $\mathrm{CN}$ and $\mathrm{HCN}$ Abundances}

Along the molecular ring around Sh104, an enhanced emission at $\sim 8 \mu \mathrm{m}$ has been detected in the MSX survey (cf. Deharveng et al. 2003). This band contains emission attributed to large molecules such as polycyclic aromatic hydrocarbons (PAHs), which can be excited in the photodissociation region (PDR) (Leger \& Puget 1984). This emission suggests that this region is experiencing significant turbulence caused by the interaction with the ionized gas. CN is chemically linked 
Table 3

Calculated abundances of $\mathrm{CN}$ and $\mathrm{HCN}$

\begin{tabular}{ccccc}
\hline $\begin{array}{c}\text { Offset }^{\mathrm{a}} \\
\left({ }^{\prime},{ }^{\prime}\right)\end{array}$ & $\begin{array}{c}\mathrm{T}_{\mathrm{ex}}(\mathrm{CN} / \mathrm{HCN})^{\mathrm{b}} \\
(\mathrm{K})\end{array}$ & $\begin{array}{c}\mathrm{N}(\mathrm{CN}) \\
\left(\mathrm{cm}^{-2}\right)\end{array}$ & $\begin{array}{c}\mathrm{N}(\mathrm{HCN}) \\
\left(\mathrm{cm}^{-2}\right)\end{array}$ & $\mathrm{CN} / \mathrm{HCN}^{\mathrm{c}}$ \\
\hline$(3,0)$ & $3.3_{-0.4}^{+0.5} / 5.1_{-0.1}^{+1.6}$ & $2.8_{-0.6}^{+1.1}(13)$ & $4.6_{-0.1}^{+0.3}(12)$ & $\sim 6$ \\
$(3,-1)$ & $4.2_{-0.4}^{+0.4} / 7.5_{-1.9}^{+0.9}$ & $3.0_{-0.4}^{+1.5}(13)$ & $6.6_{-0.1}^{+0.1}(12)$ & $\sim 5$ \\
$(2,0)$ & $4.3_{-0.9}^{+1.3} / 7.8_{-1.3}^{+1.3}$ & $1.2_{-0.4}^{+0.5}(13)$ & $4.8_{-0.1}^{+0.1}(12)$ & $\sim 3$ \\
$(2,-1)$ & $4.5_{-0.3}^{+0.4} / 8.3_{-1.0}^{+0.9}$ & $3.3_{-0.4}^{+0.4}(13)$ & $6.4_{-0.1}^{+0.1}(12)$ & $\sim 5$ \\
\hline
\end{tabular}

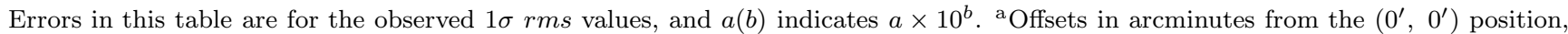
$(\alpha, \delta)_{J 2000}=\left(20^{h} 17^{m} 45^{s}, 36^{\circ} 46^{\prime} 0^{\prime \prime}\right)$. ${ }^{\mathrm{b}}$ Excitation temperature derived from the observed two transitions of each species. ${ }^{\mathrm{c}}$ Total column density ratio of $\mathrm{CN}$ and $\mathrm{HCN}$.

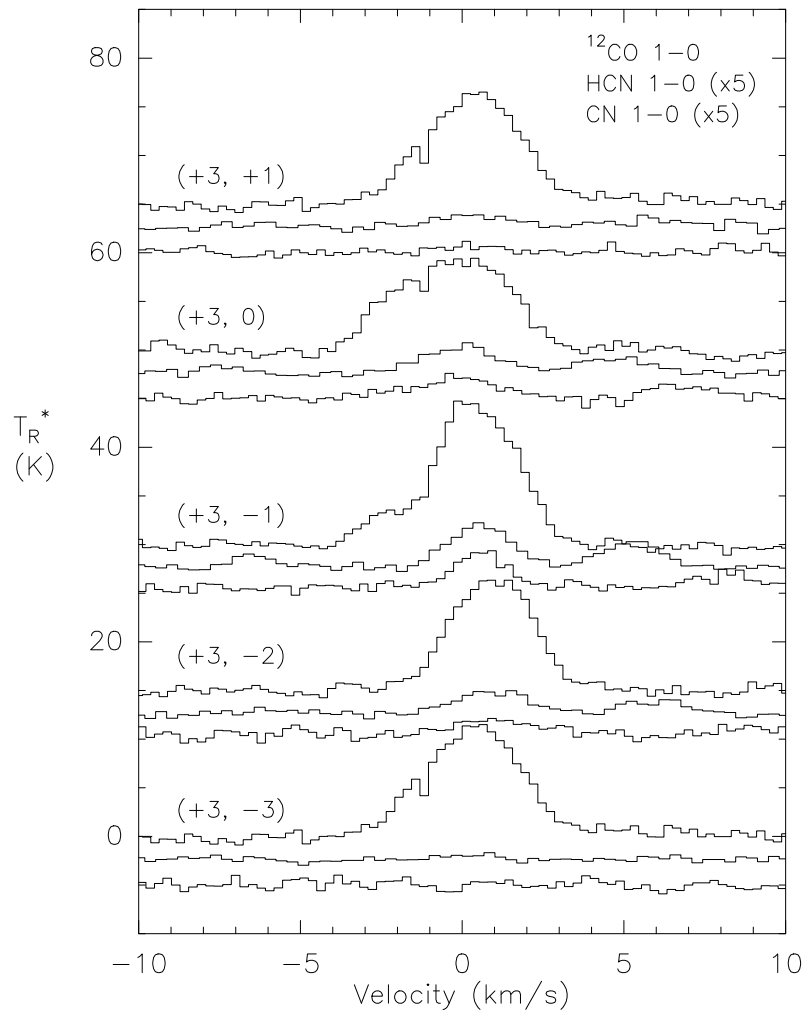

Figure 4. Sample spectra of ${ }^{12} \mathrm{CO}(1-0)$, $\mathrm{HCN}(1-0)$ and CN $(1-0)$ taken in declination at an offset $\Delta \alpha=3^{\prime}$ from a center position of $(\alpha, \delta)_{J 2000}=\left(20^{h} 17^{m} 45^{s}, 36^{\circ} 46^{\prime} 0^{\prime \prime}\right)$. The HCN and CN spectra were expanded by factor of 5 for better view.

to $\mathrm{HCN}$ and can also be formed by the direct photodissociation of HCN (Huggins et al. 1984). The CN/HCN abundance ratio has been suggested as a probe of PDRs in various objects; it is observed to be typically $\lesssim 1$ in the shielded regions of the interstellar medium, but $>1$ in PDRs with enhanced UV field (Fuente et al. 1993, 1995, 2005; Sternberg \& Dalgarno 1995; Thi et al. 2004; Jørgensen 2004). We searched for a possible enhancement of the $\mathrm{CN} / \mathrm{HCN}$ abundance ratio toward the molecular cloud embedding IRAS 20160+3636.

We derived total column densities of $\mathrm{CN}$ and $\mathrm{HCN}$ by assuming optically thin emission with an LTE condition, and results are included in Table 3. The abun- dance ratios are also included in the last column of the Table, which may suggest that this molecular cloud is being affected by UV photons from the $\mathrm{H}$ II region as shown in the $8 \mu \mathrm{m}$ data (Deharveng et al. 2003). However, the optical depths of the observed lines appear to be about 7.0 and 6.5 for the $\mathrm{CN} \mathrm{F}=5 / 2-3 / 2$ and $\mathrm{HCN}$ $\mathrm{F}=2-1$ lines toward the dense core by applying the hyperfine ratios $(\mathrm{F}=5 / 2-3 / 2 \& 3 / 2-1 / 2$ for $\mathrm{CN}$ and $\mathrm{F}=2-1$ \& 0-1 for HCN) to the gaussian fit of the observed lines. Therefore the assumption of optically thin emission can lead to a significant underestimate of the abundances of these species. However, we derived similar abundance ratios all over the observed region, where the optical depth is much less than that at the dense core. Observations of the rare isotope species of $\mathrm{CN}$ and $\mathrm{HCN}$ with higher spatial resolution are certainly necessary for further discussion on this ratio. We think that the $\mathrm{CN} / \mathrm{HCN}$ abundance ratio, if obtained with proper accuracy, will play as an important probe in understanding the collect and collapse process better.

\section{SUMmary}

We have investigated the physical parameters of the fragmented molecular cloud and the UC H II region associated with Sh104. This source is located in the eastern periphery of the gas ring shaped by the expansion of the diffuse $\mathrm{H}$ II region, which may have resulted from the "collect and collapse" process.

Comparison between our high-resolution and lowresolution radio continuum images shows that the UC H II region associated with IRAS $20160+3636$ is not physically related to the diffuse extended radio continuum emission. This suggests that it might be a bona-fide UC H II region and younger than most other UC H II regions, which usually have extended envelopes. Most derived parameters are similar to the typical values, except for a significantly smaller electron density and emission, which may be an optical depth effect.

The molecular cloud in the eastern periphery, containing IRAS $20160+3636$, is well isolated from the background or ambient emission with a roughly circular shape. The total gas mass was found to be about $(1-3) \times 10^{3} \mathrm{M}_{\odot}$ from the molecular line observations. From the $\mathrm{CN}$ and $\mathrm{HCN}$ line observations with the ARO telescopes (12m and SMT), we derived abundance ra- 
tios, $\mathrm{CN} / \mathrm{HCN}$, greater than 1 over the whole cloud, which may suggest that the whole cloud is affected by UV photons as shown in the MSX $8.3 \mu \mathrm{m}$ observations (cf., Deharveng et al. 2003). But our data suffer large optical depths, and higher spatial resolution observations for rare isotope species for $\mathrm{CN}$ and $\mathrm{HCN}$ are required to further discuss on this ratio. If the $\mathrm{CN} / \mathrm{HCN}$ abundance ratio is estimated with proper accuracy, it may be an important probe to understand the collect and collapse process.

\section{REFERENCES}

Burgh, E. B., France, K., \& McCandliss, S. R. 2007, Direct Measurement of the Ratio of Carbon Monoxide to Molecular Hydrogen in the Diffuse Interstellar Medium, ApJ, 658,446

Condon, J. J., Cotton, W. D., Greisen, E. W., Yin, Q. F., Perley, R. A., Taylor, G. B., \& Broderick, J. J. 1998, The NRAO VLA Sky Survey, AJ, 115, 1693

Crampton, D., Georgelin, Y. M., \& Georgelin, Y. P. 1978, First Optical Detection of W51 and Observations of New H II Regions and Exciting Stars, A\&A, 66, 1

Deharveng, L., Lefloch, B., Zavagno, A., Caplan, J., Whitworth, A. P., Nadeau, D., \& Martín, S. 2003, Triggered Massive-Star Formation at the Border of the H II Region Sh 104, A\&A, 408, L25

Deharveng, L., Zavagno, A., \& Caplan, J. 2005, Triggered Massive-Star Formation on the Borders of Galactic H II Regions, A\&A, 433, 565

Ellingsen, S. P., Shabala, S. S., \& Kurtz, S. E. 2005, Extended Emission Associated with Young HII Regions, MNRAS, 357, 1003

Elmegreen, B. G., \& Lada, C. J. 1977, Sequential Formation of Subgroups in OB Associations, ApJ, 214, 725

Frerking, M., Langer, L., \& Wilson, R. 1982, The Relationship between Carbon Monoxide Abundance and Visual Extinction in Interstellar Clouds, ApJ, 262, 590

Fuente, A., Martín-Pintado, J., Cerncharo, J., \& Bachiller, R. 1993, A Chemical Study of the Photodissociation Region NGC 7023, A\&A, 276, 473

Fuente, A., Martín-Pintado, J., \& Gaume, R. 1995, HighDensity CN Filaments in NGC 2023, ApJ, 442, L33

Fuente, A., Garcia-Burillo, S., Gerin, M., Teyssier, D., Usero, A., Rizzo, J. R., \& de Vicente, P. 2005, Photon-Dominated Chemistry in the Nucleus of M82: Widespread HOC+ Emission in the Inner 650 Parsec Disk ApJ, 616, L155

Georgelin, Y. M., Georgelin, Y. P., \& Roux, S. 1973, Observations de Nouvelles Regions HII Galactiques et d'etoiles Excitatrices, A\&A, 25, 337

Gomez, Y., Rodriguez, L. F., Garay, G., \& Moran, J. M. 1991, The Dense Molecular Envelope around the Compact H II Region G5.89 - 0.39 (W28 A2), ApJ, 377, 519

Harjunpää, P., Lehtinen, K., \& Haikala, L. K. 2004, The
Relationship of CO Abundance to Extinction and N(H2): Observations of Globules and the Dependence on Star Formation Activity, A\&A, 421, 1087

Huggins, P. J., Glassgold, A. E., \& Morris, M. 1984, CN and $\mathrm{C} 2 \mathrm{H}$ in IRC +10216, ApJ, 279, 284

Israël, F. P. 1977, Aperture Synthesis Observations of Galactic H II Regions. VI - Several Isolated H II Regions, A\&A, 60, 233

Jørgensen, J. K. 2004, Imaging Chemical Differentiation around the Low-Mass Protostar L483-mm, A\&A, 424, 589

Kim, K.-T., \& Koo, B.-C. 2001, Radio Continuum and Recombination Line Study of Ultracompact H II Regions with Extended Envelopes, ApJ, 549, 979

Kurtz, S., Churchwell, E., \& Wood, D. O. S. 1994, Ultracompact H II Regions. 2: New High-Resolution Radio Images, ApJS, 91, 659

Kurtz, S. E., Watson, A. M., Hofner, P., \& Otte, B. 1999, Ultracompact H II Regions with Extended RadioContinuum Emission, ApJ, 514, 232

Lahulla, J. F. 1985, UBVRI Photometry of Stars in Several H II Regions, A\&A Suppl. Ser., 61, 537

Leger, A., \& Puget, J. L. 1984, Identification of the 'Unidentified' IR Emission Features of Interstellar Dust?, A\&A, 137, L5

Mezger, P. G., \& Henderson, A. P. 1967, Galactic H II Regions. I. Observations of Their Continuum Radiation at the Frequency $5 \mathrm{GHz}, \mathrm{ApJ}, 147,471$

Pérez-Beaupuits, J. P., Aalto, S., \& Gerebro, H. 2007, HNC, HCN and CN in Seyfert Galaxies, A\&A, 476, 177

Reifenstein III, E. C., Wilson, T. L., Burke, B. F., Mezger, P. G., \& Altenhoff, W. J. 1970, A Survey of H 1O9x Recombination Line Emission in Galactic HN Regions of the Northern Sky, A\&A, 4, 357

Rubin, R. H. 1968, A Discussion of the Sizes and Excitation of H II Regions, ApJ, 154, 391

Steer, D., Dwedney, P., \& Ito, M. 1984, Enhancements to the Deconvolution Algorithm 'CLEAN', A\&A, 137, 159

Sternberg, A., \& Dalgarno, A. 1995, Chemistry in Dense Photon-dominated Regions, ApJS, 99, 565

Swarup, G., Ananthakrishnan, S., Kapahi, V. K., Rao, A. P., Subrahmanya, C. R., \& Kulkarni, V. K. 1991, The Giant Metre-Wave Radio Telescope, Current Science, 60, 95

Thi, W.-F., van Zadelhoff, G.-J., \& E. F. van Dishoeck, E. F. 2004, Organic Molecules in Protoplanetary Disks around T Tauri and Herbig Ae Stars, A\&A, 425, 955

Whitworth, A. P., Bhattal, A. S., Chapman, S. J., Disney, M. J., \& Turner, J. A. 1994, The Preferential Formation of High-Mass Stars in Shocked Interstellar Gas Layers, MNRAS, 268, 291

Wood, D. O. S., \& Churchwell, E. 1989, Massive Stars Embedded in Molecular Clouds - Their Population and Distribution in the Galaxy, ApJ, 340, 265 